\title{
Highly efficient synthesis of 7-aryl-pyrano[3,4-c]pyrazolo $[4,3-f]$ quinoline derivatives catalyzed by iodine
}

\author{
Wei Wang, Yu-Ling Li, and Xiang-Shan Wang * \\ School of Chemistry and Chemical Engineering, Jiangsu Key Laboratory of Green Synthetic \\ Chemistry for Functional Materials, Jiangsu Normal University, Xuzhou, Jiangsu 221116, \\ P.R. China \\ E-mail:xswang1974@yahoo.com
}

\begin{abstract}
A mild and efficient method for the synthesis of 7-arylpyrano[3,4-c]pyrazolo[3,4-f]quinoline derivatives via a three-component reaction of aromatic aldehyde, $1 H$-Indazol-5-amine and tetrahydropyran-4-one catalyzed by iodine is described. This new procedure has the advantages of mild reaction condition, high yields, one-pot and metal-free catalyst.
\end{abstract}

Keywords: Three-component reaction, $1 H$-indazol-5-amine, pyrazolo[3,4-f]quinoline, iodine

\section{Introduction}

Pyranoquinoline derivatives are found to possess a wide spectrum of biological activities, such as psychotropic, anti-allergenic, anti-inflammatory and estrogenic activities. ${ }^{1}$ Pyrazoloquinoline derivatives are also an important class of heterocylcles due to their promising materials for optoelectronic applications. ${ }^{2}$ In addition, they are reported to possess anti-leishmanial and antimicrobial activities. ${ }^{3}$ Some of them are used as modulators of cytokine biosynthesis for treatment of viral and neoplastic diseases, ${ }^{4}$ and immuno modulators for inducing cytokine biosynthesis in animals. ${ }^{5}$

Although a number of useful synthetic procedures to prepare pyranoquinoline ${ }^{6}$ and pyrazoloquinoline $^{7}$ derivatives have been developed, to the best of our acknowledge, there is no literature about the synthesis of fused heterocycle containing both pyran, pyrazole and quinoline rings, this novel skeleton may posses potential bioactive for screening. Thus, simple and efficient method to synthesize pyranopyrazoloquinolines would be attractive,

Over the past few years, molecular iodine $\left(\mathrm{I}_{2}\right)$ has emerged as a powerful catalyst for various organic transformations due to several advantages such as its inexpensive, nontoxic, and ecofriendly nature. ${ }^{8}$ As a continuation of our research devoted to the development of new methods for the preparation of heterocycles via multi-component reactions catalyzed by iodine, ${ }^{9}$ herein, 
we would like to report the synthesis of 7-aryl-pyrano[3,4-c]pyrazolo[3,4-f]quinoline derivatives by a reaction of aromatic aldehyde, $1 H$-indazol-5-amine and tetrahydropyran-4-one in THF catalyzed by iodine.

\section{Results and Discussion}

Treatment of aromatic aldehyde 1a-1, $1 H$-indazol-5-amine $\mathbf{2}$ and tetrahydropyran-4-one $\mathbf{3}$ in THF in the presence of $5 \mathrm{~mol} \%$ iodine at reflux condition afforded the corresponding 7-arylpyrano[3,4-c]pyrazolo[3,4-f]quinoline derivatives 4a-l in high yields (Scheme 1).

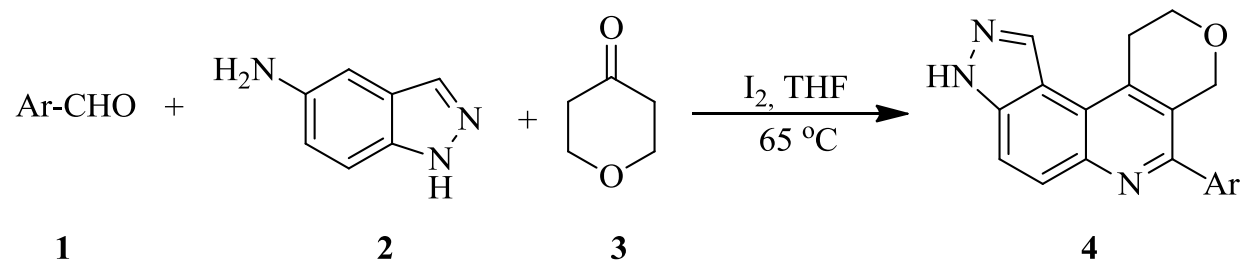

Scheme 1. The reaction of 1a-1, 2 and cyclopentanone 3.

Table 1. Synthetic results of $\mathbf{4 a}$ under different reaction conditions ${ }^{a}$

\begin{tabular}{llllll}
\hline Entry & Temp. $\left({ }^{\circ} \mathrm{C}\right)$ & Cat. $($ mol \%) & Solvent & Time (h) & Yields $(\%)^{b}$ \\
\hline 1 & r.t. & - & THF & 24 & 0 \\
2 & Reflux & - & THF & 24 & 0 \\
3 & r.t. & $\mathrm{I}_{2}(5)$ & $\mathrm{THF}$ & 24 & trace \\
4 & 50 & $\mathrm{I}_{2}(5)$ & $\mathrm{THF}$ & 24 & 84 \\
5 & Reflux & $\mathrm{I}_{2}(5)$ & $\mathrm{THF}$ & 12 & 93 \\
6 & Reflux & $\mathrm{I}_{2}(10)$ & $\mathrm{THF}$ & 12 & 92 \\
7 & Reflux & $\mathrm{I}_{2}(20)$ & $\mathrm{THF}$ & 12 & 93 \\
8 & Reflux & $\mathrm{I}_{2}(5)$ & $\mathrm{CH}{ }_{3} \mathrm{CN}$ & 12 & 82 \\
9 & Reflux & $\mathrm{I}_{2}(5)$ & $\mathrm{Benzene}$ & 10 & 78 \\
10 & 80 & $\mathrm{I}_{2}(5)$ & $\mathrm{DMF}$ & 10 & 85 \\
11 & Reflux & $\mathrm{I}_{2}(5)$ & $\mathrm{CHCl}$ & 12 & 77 \\
12 & Reflux & $\mathrm{TsOH}_{3}(5)$ & $\mathrm{THF}$ & 10 & 78 \\
13 & Reflux & $\mathrm{ZnCl}_{2}(5)$ & $\mathrm{THF}$ & 18 & 0 \\
14 & Reflux & $\mathrm{Yb}_{2}(\mathrm{OTf})_{3}(5)$ & $\mathrm{THF}$ & 12 & 69 \\
15 & Reflux & $\mathrm{Sc}_{(\mathrm{OTf})}(5)$ & $\mathrm{THF}$ & 10 & 82 \\
\hline
\end{tabular}

${ }^{a}$ Reagents and conditions: 2-chlorobenzaldehyde 1a (0.281 g, $\left.2.0 \mathrm{mmol}\right), 2$ (0.266 g, $\left.2.0 \mathrm{mmol}\right)$, $3(0.200 \mathrm{~g}, 2.0 \mathrm{mmol})$, solvent $(10 \mathrm{~mL}) .{ }^{b}$ Isolated yields. 
Using the conversion of 2-chlorobenzaldehyde 1a, $\mathbf{2}$ and $\mathbf{3}$ as a model, several parameters were explored as shown in Table 1. The 4a was not detected by TLC at room temperature and reflux in the absence of iodine (Table 1, Entries 1 and 2), and much greater in the presence of various quantities of the catalyst $\left(\mathrm{I}_{2}\right)$, reaching a maximum of $93 \%$ yield with 5 mol\% iodine (Table 1, entries 5-7). The yield of 4a was also dependent on temperature (entries 3 5), proceeding smoothly at reflux in high yield. Different solvents were also tested, and THF appeared to be the best medium for this transformation (entry 5 vs. 8-11). In addition, other Lewis acids, such as $\mathrm{TsOH}, \mathrm{ZnCl}_{2}, \mathrm{Yb}(\mathrm{OTf})_{3}$ and $\mathrm{Sc}(\mathrm{OTf})_{3}$, were selected as catalysts to this reaction in $5 \mathrm{~mol} \%$, they all gave slightly lower yields.

These optimized conditions were applied to the conversion of various kinds of aromatic aldehydes 1a-l into the corresponding 7-aryl-pyrano[3,4-c]pyrazolo[3,4-f]quinoline analogues 4a-l. Reactions using aldehydes containing electron-withdrawing groups (such as halide) or electron-donating groups (such as alkyl and alkoxy group) all proceeded smoothly within a few hours, giving 4a-l in high yields. It should be noted that only 7-aryl-pyrano[3,4-c]pyrazolo[3,4$f$ ]quinolines were obtained in high region-chemistry. The best reason is that the 4-position is not only the ortho-position to amino group, but also the $\alpha$-position of the benzene ring in $1 H$ indazol-5-amine, which is more active than 6-position.

Table 2. Synthetic results of $\mathbf{4 a - 1}$ catalyzed by iodine ${ }^{a}$

\begin{tabular}{llccc}
\hline Entry & $\mathrm{Ar}$ & time $(\mathrm{h})$ & products & yields $(\%)^{b}$ \\
\hline 1 & $2-\mathrm{ClC}_{6} \mathrm{H}_{4}$ & 12 & $\mathbf{4 a}$ & 93 \\
2 & $4-\mathrm{ClC}_{6} \mathrm{H}_{4}$ & 14 & $\mathbf{4 b}$ & 90 \\
3 & $4-\mathrm{BrC}_{6} \mathrm{H}_{4}$ & 14 & $\mathbf{4 c}$ & 92 \\
4 & $4-\mathrm{MeOC}_{6} \mathrm{H}_{4}$ & 18 & $\mathbf{4 d}$ & 87 \\
5 & $4-\mathrm{MeC}_{6} \mathrm{H}_{4}$ & 14 & $\mathbf{4 e}$ & 83 \\
6 & $3-\mathrm{ClC}_{6} \mathrm{H}_{4}$ & 14 & $\mathbf{4 f}$ & 90 \\
7 & $4-\mathrm{FC}_{6} \mathrm{H}_{4}$ & 10 & $\mathbf{4 g}$ & 88 \\
8 & $3,4-\mathrm{Cl}_{2} \mathrm{C}_{6} \mathrm{H}_{3}$ & 12 & $\mathbf{4 h}$ & 89 \\
9 & $3-\mathrm{BrC}_{6} \mathrm{H}_{4}$ & 14 & $\mathbf{4 i}$ & 90 \\
10 & $3,4-\left(\mathrm{MeO}_{2} \mathrm{C}_{6} \mathrm{H}_{3}\right.$ & 16 & $\mathbf{4 j}$ & 84 \\
11 & $3,4-\mathrm{Me}_{2} \mathrm{C}_{6} \mathrm{H}_{3}$ & 18 & $\mathbf{4 k}$ & 87 \\
12 & Piperonyl & 16 & $\mathbf{4 l}$ & 90 \\
\hline
\end{tabular}

${ }^{\mathrm{a}}$ Reagents and conditions: benzaldehyde 1a-l $(2.0 \mathrm{mmol}), 2$ (0.266 g, $\left.2.0 \mathrm{mmol}\right), 3$ (0.200 g, 2.0 $\mathrm{mmol}), \mathrm{THF}(10 \mathrm{~mL}) .{ }^{\mathrm{b}}$ Isolated yields.

According to the literatures, ${ }^{10}$ we think that iodine catalyzes the reaction as a mild Lewis acid. The mechanism was tentatively proposed as shown in Scheme 2. In the presence of iodine, tetrahydropyran-4-one is in equilibrium with its enol form. ${ }^{10 \mathrm{~b}}$ The Schiff base I may be formed by the reaction of aromatic aldehyde and $1 H$-indazol-5-amine firstly. And then imino-Diels- 
Alder reaction between the iodine-activated Schiff base II and enol takes place selectively to form the intermediate III for its stability. The dehydration of III results in dihydro pyrano[3,4c]pyrazolo[3,4-f]quinoline $\mathbf{I V}$, which is further oxidized by air to afford aromatized final products 4 .

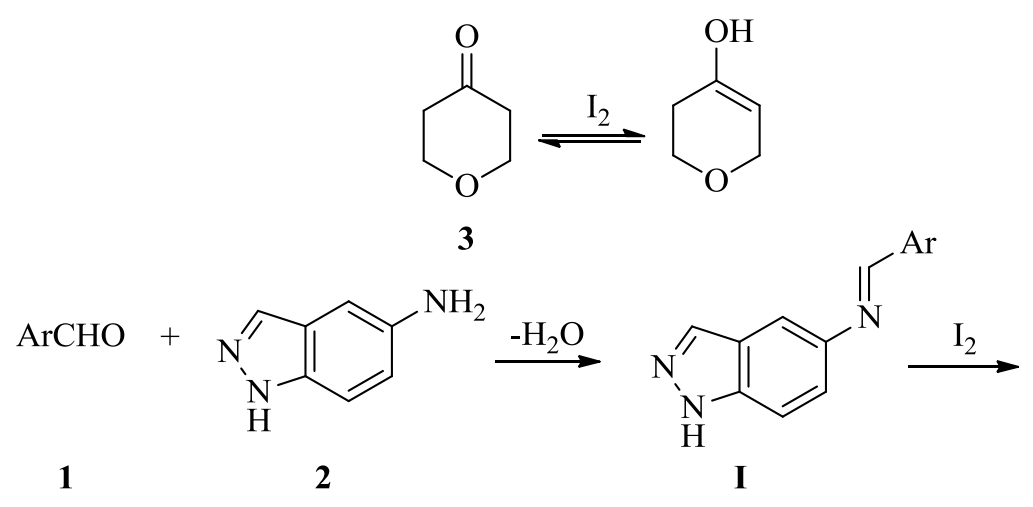<smiles>[Al]=CNc1ccc2[nH]ncc2c1</smiles>

II

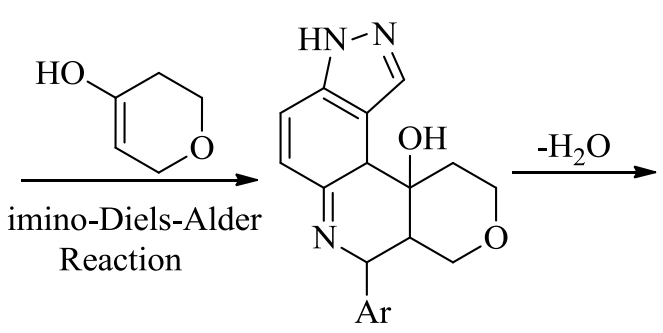

III



IV

Scheme 2. The possible mechanism for the formation of products 4 .

\section{Conclusions}

In conclusion, we found a mild and efficient method for the synthesis of 7-aryl-pyrano[3,4c]pyrazolo[3,4-f]quinoline derivatives via three-component reactions of aromatic aldehyde, $1 H$ indazol-5-amine and tetrahydropyran-4-one catalyzed by iodine. The features of this procedure are mild reaction conditions, high yields, operational simplicity, one-pot and metal-free catalyst.

\section{Experimental Section}

General. Melting points were determined in open capillaries and are uncorrected. IR spectra were recorded on a Tensor 27 spectrometer in $\mathrm{KBr}$ pellet. ${ }^{1} \mathrm{H}$ NMR spectra and ${ }^{13} \mathrm{C}$ NMR was obtained from a solution in $\mathrm{CDCl}_{3}$ or DMSO- $d_{6}$ with $\mathrm{Me}_{4} \mathrm{Si}$ as internal standard using a Bruker400 spectrometer. HRMS analyses were carried out using a Bruker-micro-TOF-Q-MS analyzer. 
General procedure for the syntheses of 7-aryl-pyrano[3,4-c]pyrazolo[3,4-f]quinoline derivatives $4 a-1$

A dry $50 \mathrm{~mL}$ flask was charged with aromatic aldehyde $(2.0 \mathrm{mmol}), 1 H$-indazol-5-amine $(0.266$ $\mathrm{g}, 2.0 \mathrm{mmol})$, tetrahydropyran-4-one $(0.200 \mathrm{~g}, 2.0 \mathrm{mmol}), \mathrm{I}_{2}(0.026 \mathrm{~g}, 0.1 \mathrm{mmol})$ and THF (10 $\mathrm{mL})$. The reaction mixture was stirred at reflux for 10-18 h. After completion of the reaction as indicated by TLC, a little DMF was added to the mixture until the yellow precipitate was dissolved. The generated crystals were collected by filtration to give $\mathbf{4}$ when the mixture was cooled to room temperature.

7-(2-Chlorophenyl)-3,8,10,11-tetrahydropyrano[3,4-c]pyrazolo[4,3-f]quinoline (4a). Mp 281$283{ }^{\circ} \mathrm{C} ;{ }^{1} \mathrm{H}$ NMR (DMSO- $\left.d_{6}, 400 \mathrm{MHz}\right): \delta_{\mathrm{H}} 3.43 \sim 3.46\left(\mathrm{~m}, 2 \mathrm{H}, \mathrm{CH}_{2}\right), 4.01 \sim 4.13\left(\mathrm{~m}, 2 \mathrm{H}, \mathrm{CH}_{2}\right)$, $4.51\left(\mathrm{~s}, 2 \mathrm{H}, \mathrm{CH}_{2}\right), 7.45 \sim 7.56(\mathrm{~m}, 3 \mathrm{H}, \mathrm{ArH}), 7.63(\mathrm{~d}, J 7.6 \mathrm{~Hz}, 1 \mathrm{H}, \mathrm{ArH}), 7.88(\mathrm{~d}, J 9.2 \mathrm{~Hz}, 1 \mathrm{H}$, ArH), 7.95 (d, $J 8.8 \mathrm{~Hz}, 1 \mathrm{H}, \mathrm{ArH}), 8.61(\mathrm{~s}, 1 \mathrm{H}, \mathrm{CH}), 13.76(\mathrm{~s}, 1 \mathrm{H}, \mathrm{NH}) .{ }^{13} \mathrm{C}$ NMR (DMSO- $d_{6}$, $100 \mathrm{MHz}): \delta_{\mathrm{C}} 28.4,64.3,65.9,113.2,115.2,116.3,121.7,127.8,128.0,129.4,129.9,130.8$, 132.0, 135.9, 138.2, 138.7, 139.3, 144.0, 151.8. IR (KBr): v3207, 3150, 3113, 3054, 2892, 1662, 1608, 1574, 1531, 1478, 1435, 1368, 1321, 1234, 1180, 1162, 1079, 1051, 980, 886, 844, 790, $708 \mathrm{~cm}^{-1}$. HRMS (ESI, $\mathrm{m} / z$ ): Calcd for $\mathrm{C}_{19} \mathrm{H}_{15} \mathrm{ClN}_{3} \mathrm{O}[\mathrm{M}+\mathrm{H}]^{+} 336.0904$, found 336.0928 .

7-(4-Chlorophenyl)-3,8,10,11-tetrahydropyrano[3,4-c]pyrazolo[4,3-f]quinoline (4b). Mp > $300{ }^{\circ} \mathrm{C} ;{ }^{1} \mathrm{H}$ NMR (DMSO- $\left.d_{6}, 400 \mathrm{MHz}\right): \delta_{\mathrm{H}} 3.43\left(\mathrm{~s}, 2 \mathrm{H}, \mathrm{CH}_{2}\right), 4.15 \sim 4.18\left(\mathrm{~m}, 2 \mathrm{H}, \mathrm{CH}_{2}\right), 4.80(\mathrm{~s}$, $\left.2 \mathrm{H}, \mathrm{CH}_{2}\right), 7.57$ (d, J 8.4 Hz, 2H, ArH), $7.64(\mathrm{~d}, J 8.4 \mathrm{~Hz}, 2 \mathrm{H}, \mathrm{ArH}), 7.88 \sim 7.96(\mathrm{~m}, 2 \mathrm{H}, \operatorname{ArH})$, $8.59(\mathrm{~s}, 1 \mathrm{H}, \mathrm{CH}), 13.72(\mathrm{~s}, 1 \mathrm{H}, \mathrm{NH}) .{ }^{13} \mathrm{C}$ NMR (DMSO- $\left.d_{6}, 100 \mathrm{MHz}\right): 28.2,63.9,66.3,114.7$, $115.7,120.8,127.1,128.3,129.0,130.7,133.2,135.5,138.0,138.2,139.1,143.6,152.0$. IR (KBr): v 3195, 3156, 3105, 3047, 2880, 1588, 1564, 1529, 1493, 1431, 1400, 1353, 1323, 1241, $1119,1093,1017,978,952,886,823,790,739 \mathrm{~cm}^{-1}$. HRMS (ESI, $\left.\mathrm{m} / z\right)$ : Calcd for $\mathrm{C}_{19} \mathrm{H}_{15} \mathrm{ClN}_{3} \mathrm{O}$ $[\mathrm{M}+\mathrm{H}]^{+} 336.0904$, found 336.0897 .

7-(4-Bromophenyl)-3,8,10,11-tetrahydropyrano[3,4-c]pyrazolo[4,3-f]quinoline (4c). Mp > $300{ }^{\circ} \mathrm{C} ;{ }^{1} \mathrm{H}$ NMR (DMSO-d, $\left.400 \mathrm{MHz}\right): \delta_{\mathrm{H}} 3.42\left(\mathrm{~s}, 2 \mathrm{H}, \mathrm{CH}_{2}\right), 4.16\left(\mathrm{t}, J 5.2 \mathrm{~Hz}, 2 \mathrm{H}, \mathrm{CH}_{2}\right), 4.81$ (s, 2H, $\left.\mathrm{CH}_{2}\right), 7.57(\mathrm{~d}, J 8.0 \mathrm{~Hz}, 2 \mathrm{H}, \mathrm{ArH}), 7.72(\mathrm{~d}, J 8.0 \mathrm{~Hz}, 2 \mathrm{H}, \mathrm{ArH}), 7.90 \sim 7.95$ (m, 2H, ArH), $8.60(\mathrm{~s}, 1 \mathrm{H}, \mathrm{CH}), 13.74(\mathrm{~s}, 1 \mathrm{H}, \mathrm{NH}) .{ }^{13} \mathrm{C}$ NMR (DMSO- $\left.d_{6}, 100 \mathrm{MHz}\right): \delta_{\mathrm{C}} 28.7,64.4,66.8,101.4$, 115.3, 116.2, 122.4, 127.6, 129.5, 131.5, 131.7, 135.96, 136.03, 138.9, 139.7, 144.2, 153.6. IR (KBr): v 3190, 3153, 3103, 3047, 2887, 1665, 1587, 1528, 1489, 1395, 1353, 1322, 1240, 1136, 1100, 1072, 1012, 977, 952, 866, 849, 821, 790, $737 \mathrm{~cm}^{-1}$. HRMS (ESI, $\left.\mathrm{m} / z\right)$ : Calcd for $\mathrm{C}_{19} \mathrm{H}_{15} \mathrm{BrN}_{3} \mathrm{O}[\mathrm{M}+\mathrm{H}]^{+} 380.0398$, found 380.0394 .

7-(4-Methoxyphenyl)-3,8,10,11-tetrahydropyrano[3,4-c]pyrazolo[4,3-f]quinoline (4d). Mp 267-269 ${ }^{\circ} \mathrm{C} ;{ }^{1} \mathrm{H}$ NMR (DMSO- $\left.d_{6}, 400 \mathrm{MHz}\right): \delta_{\mathrm{H}} 3.42\left(\mathrm{~s}, 2 \mathrm{H}, \mathrm{CH}_{2}\right), 3.84\left(\mathrm{~s}, 3 \mathrm{H}, \mathrm{OCH}_{3}\right)$, 4.15 4.18 (m, 2H, CH$), 4.81\left(\mathrm{~s}, 2 \mathrm{H}, \mathrm{CH}_{2}\right), 7.06$ (d, J $\left.8.8 \mathrm{~Hz}, 2 \mathrm{H}, \mathrm{ArH}\right), 7.55$ (d, J 8.8 Hz, 2H, $\mathrm{ArH}), 7.87 \sim 7.93(\mathrm{~m}, 2 \mathrm{H}, \mathrm{ArH}), 8.58(\mathrm{~s}, 1 \mathrm{H}, \mathrm{CH}), 13.71(\mathrm{~s}, 1 \mathrm{H}, \mathrm{NH}) .{ }^{13} \mathrm{C}$ NMR (DMSO-d, 100 $\mathrm{MHz}): \delta_{\mathrm{C}} 28.2,55.1,63.9,66.6,113.5,114.5,115.8,120.4,127.1,129.1,130.2,131.6,135.4$, 138.1, 138.8, 143.7, 153.1, 159.3. IR (KBr): v 3251, 2930, 2860, 1608, 1590, 1561, 1509, 1460, 1441, 1348, 1322, 1242, 1181, 1119, 1079, 1033, 988, 949, 889, 832, 816, 801, 787, $733 \mathrm{~cm}^{-1}$. HRMS (ESI, $m / z$ ): Calcd for $\mathrm{C}_{20} \mathrm{H}_{18} \mathrm{~N}_{3} \mathrm{O}_{2}[\mathrm{M}+\mathrm{H}]^{+} 332.1399$, found 332.1414. 
7-(4-Methylphenyl)-3,8,10,11-tetrahydropyrano[3,4-c]pyrazolo[4,3-f]quinoline (4e). Mp 281 283 ${ }^{\circ} \mathrm{C},{ }^{1} \mathrm{H}$ NMR (DMSO- $\left.d_{6}, 400 \mathrm{MHz}\right): \delta_{\mathrm{H}} 2.40\left(\mathrm{~s}, 3 \mathrm{H}, \mathrm{CH}_{3}\right), 3.42\left(\mathrm{~s}, 2 \mathrm{H}, \mathrm{CH}_{2}\right)$, 4.15 4.17 (m, 2H, $\left.\mathrm{CH}_{2}\right), 4.79$ (s, 2H, $\left.\mathrm{CH}_{2}\right), 7.32$ (d, J $\left.8.0 \mathrm{~Hz}, 2 \mathrm{H}, \mathrm{ArH}\right), 7.49$ (d, J 7.2 Hz, 2H, $\mathrm{ArH}), 7.87 \sim 7.96(\mathrm{~m}, 2 \mathrm{H}, \mathrm{ArH}), 8.58(\mathrm{~s}, 1 \mathrm{H}, \mathrm{CH}), 13.72(\mathrm{~s}, 1 \mathrm{H}, \mathrm{NH}) .{ }^{13} \mathrm{C}$ NMR (DMSO-d, 100 $\mathrm{MHz}): \delta_{\mathrm{C}} 20.8,28.2,63.9,66.5,114.5,115.8,120.6,127.1,128.66,128.75,129.1,135.4,136.5$, 137.7, 138.1, 138.8, 143.7, 153.4. IR (KBr): v 3187, 3131, 3093, 3037, 2980, 1670, 1592, 1561, 1530, 1510, 1451, 1428, 1384, 1365, 1349, 1322, 1237, 1160, 1099, 1034, 984, 938, 889, 826, $791,741 \mathrm{~cm}^{-1}$. HRMS (ESI, $m / z$ ): Calcd for $\mathrm{C}_{20} \mathrm{H}_{18} \mathrm{~N}_{3} \mathrm{O}[\mathrm{M}+\mathrm{H}]^{+} 316.1450$, found 316.1464 .

7-(3-Chlorophenyl)-3,8,10,11-tetrahydropyrano[3,4-c]pyrazolo[4,3-f]quinoline $\quad(4 \mathrm{f}) . \quad \mathrm{Mp}$ 277 279 ${ }^{\circ} \mathrm{C} ;{ }^{1} \mathrm{H}$ NMR (DMSO- $\left.d_{6}, 400 \mathrm{MHz}\right): \delta_{\mathrm{H}} 3.42\left(\mathrm{~s}, 2 \mathrm{H}, \mathrm{CH}_{2}\right), 4.15 \sim 4.18\left(\mathrm{~m}, 2 \mathrm{H}, \mathrm{CH}_{2}\right)$, 4.79 (s, 2H, $\left.\mathrm{CH}_{2}\right), 7.56$ (s, 3H, ArH), 7.67 (s, 1H, ArH), $7.88 \sim 7.95$ (m, 2H, ArH), 8.59 (s, 1H, $\mathrm{CH}), 13.75(\mathrm{~s}, 1 \mathrm{H}, \mathrm{NH}) .{ }^{13} \mathrm{C}$ NMR $\left(\mathrm{DMSO}-d_{6}, 100 \mathrm{MHz}\right): \delta_{\mathrm{C}} 28.6,64.3,66.7,115.3,116.2$, 121.5, 127.6, 127.9, 128.8, 129.1, 129.5, 130.6, 133.5, 136.0, 138.7, 139.7, 141.7, 144.1, 152.2. IR (KBr): $v$ 3272, 3163, 3038, 2958, 2852, 1669, 1595, 1556, 1531, 1475, 1417, 1357, 1320, 1298, 1237, 1094, 1033, 979, 946, 885, 836, 823, 789, 721, $706 \mathrm{~cm}^{-1}$. HRMS (ESI, m/z): Calcd for $\mathrm{C}_{19} \mathrm{H}_{15} \mathrm{ClN}_{3} \mathrm{O}[\mathrm{M}+\mathrm{H}]^{+} 336.0904$, found 336.0924.

7-(4-Fluorophenyl)-3,8,10,11-tetrahydropyrano[3,4-c]pyrazolo[4,3-f]quinoline (4g). Mp > $300{ }^{\circ} \mathrm{C} ;{ }^{1} \mathrm{H}$ NMR (DMSO- $\left.d_{6}, 400 \mathrm{MHz}\right): \delta_{\mathrm{H}} 3.42\left(\mathrm{~s}, 2 \mathrm{H}, \mathrm{CH}_{2}\right), 4.15 \sim 4.17\left(\mathrm{~m}, 2 \mathrm{H}, \mathrm{CH}_{2}\right), 4.79(\mathrm{~s}$, $\left.2 \mathrm{H}, \mathrm{CH}_{2}\right), 7.34$ (t, J 8.8 Hz, 2H, ArH), 7.64 7.67 (m, 2H, ArH), 7.87 7.94 (m, 2H, ArH), 8.59 (s, 1H, CH), 13.74 (s, 1H, NH). IR (KBr): v 3191, 3157, 3108, 3053, 2942, 2879, 1665, 1603, 1588, 1531, 1510, 1434, 1351, 1322, 1217, 1182, 1156, 1100, 1036, 979, 953, 885, 845, 789, 740 $\mathrm{cm}^{-1}$. HRMS (ESI, $\left.m / z\right)$ : Calcd for $\mathrm{C}_{19} \mathrm{H}_{15} \mathrm{FN}_{3} \mathrm{O}[\mathrm{M}+\mathrm{H}]^{+} 320.1199$, found 320.1205.

7-(3,4-Dichlorophenyl)-3,8,10,11-tetrahydropyrano[3,4-c]pyrazolo[4,3-f]quinoline (4h). Mp $>300{ }^{\circ} \mathrm{C} ;{ }^{1} \mathrm{H}$ NMR $\left(\mathrm{DMSO}-d_{6}, 400 \mathrm{MHz}\right): \delta_{\mathrm{H}} 3.44\left(\mathrm{~s}, 2 \mathrm{H}, \mathrm{CH}_{2}\right), 4.17\left(\mathrm{t}, J 4.2 \mathrm{~Hz}, 2 \mathrm{H}, \mathrm{CH}_{2}\right)$, $4.84\left(\mathrm{~s}, 2 \mathrm{H}, \mathrm{CH}_{2}\right), 7.62(\mathrm{~d}, J 8.8 \mathrm{~Hz}, 1 \mathrm{H}, \mathrm{ArH}), 7.80(\mathrm{~d}, J 8.4 \mathrm{~Hz}, 1 \mathrm{H}, \mathrm{ArH}), 7.89 \sim 7.96$ (m, 3H, ArH), 8.61(s, 1H, CH), 13.77 (s, 1H, NH). IR (KBr): v 3187, 3147, 3101, 2992, 2951, 2877, 1670, 1585, 1526, 1471, 1434, 1391, 1349, 1312, 1280, 1241, 1118, 1140, 1105, 1033, 978, 952, 884, 847, 820, 788, 747, $705 \mathrm{~cm}^{-1}$. HRMS (ESI, m/z): Calcd for $\mathrm{C}_{19} \mathrm{H}_{14} \mathrm{Cl}_{2} \mathrm{~N}_{3} \mathrm{O}[\mathrm{M}+\mathrm{H}]^{+}$ 370.0514 , found 370.0515 .

7-(3-Bromophenyl)-3,8,10,11-tetrahydropyrano[3,4-c]pyrazolo[4,3-f]quinoline (4i). Mp 288 290 ${ }^{\circ} \mathrm{C} ;{ }^{1} \mathrm{H}$ NMR (DMSO- $d_{6}, 400 \mathrm{MHz}$ ): $\delta_{\mathrm{H}} 3.42\left(\mathrm{~s}, 2 \mathrm{H}, \mathrm{CH}_{2}\right), 4.17\left(\mathrm{t}, J 9.6 \mathrm{~Hz}, 2 \mathrm{H}, \mathrm{CH}_{2}\right)$, $4.81\left(\mathrm{~s}, 2 \mathrm{H}, \mathrm{CH}_{2}\right), 7.48(\mathrm{t}, J 8.0 \mathrm{~Hz}, 1 \mathrm{H}, \mathrm{ArH}), 7.61$ (d, J $\left.8.0 \mathrm{~Hz}, 1 \mathrm{H}, \mathrm{ArH}\right), 7.70$ (d, J 8.0 Hz, 1H, ArH), 7.80 (s, 1H, ArH), 7.89 7.96 (m, 2H, ArH), 8.60 (s, 1H, CH), 13.75 (s, 1H, NH). IR (KBr): v 3193, 3152, 3104, 3050, 2953, 2874, 1664, 1587, 558, 1528, 1475, 1431, 1348, 1318, 1238, 1180, 1119, 1101, 1073, 979, 952, 86, 843, 788, 736, $707 \mathrm{~cm}^{-1}$. HRMS (ESI, m/z): Calcd for $\mathrm{C}_{19} \mathrm{H}_{15} \mathrm{BrN}_{3} \mathrm{O}[\mathrm{M}+\mathrm{H}]^{+} 380.0398$, found 380.0401 .

7-(3,4-Dimethoxyphenyl)-3,8,10,11-tetrahydropyrano[3,4-c]pyrazolo[4,3-f]quinoline (4j). Mp 239 241 ${ }^{\circ} \mathrm{C} ;{ }^{1} \mathrm{H}$ NMR (DMSO- $\left.d_{6}, 400 \mathrm{MHz}\right): \delta_{\mathrm{H}} 3.17 \sim 3.26(\mathrm{~m}, 1 \mathrm{H}, \mathrm{CH}), 3.72(\mathrm{~s}, 3 \mathrm{H}$, $\left.\mathrm{OCH}_{3}\right), 3.78\left(\mathrm{~s}, 3 \mathrm{H}, \mathrm{OCH}_{3}\right), 3.92(\mathrm{~d}, J 11.2 \mathrm{~Hz}, 1 \mathrm{H}, \mathrm{CH}), 4.27 \sim 4.39\left(\mathrm{~m}, 2 \mathrm{H}, \mathrm{CH}_{2}\right), 5.99(\mathrm{~s}, 1 \mathrm{H}$, $\mathrm{CH}), 6.39$ (s, 1H, CH), 6.84 (d, J $8.8 \mathrm{~Hz}, 1 \mathrm{H}, \mathrm{ArH}), 6.91 \sim 6.92(\mathrm{~m}, 2 \mathrm{H}, \mathrm{ArH}), 7.08(\mathrm{~s}, 1 \mathrm{H}, \operatorname{ArH})$, 
7.24 (d, J $8.8 \mathrm{~Hz}, 1 \mathrm{H}, \mathrm{ArH}), 8.18$ (s, 1H, CH), 12.86 (s, 1H, NH). IR (KBr): v3351, 3262, 2987, 2966, 2869, 2828, 1634, 1591, 1515, 1491, 1460, 1420, 1387, 1372, 1334, 1271, 1232, 1144, 1024, 943, 888, 825, 805, $692 \mathrm{~cm}^{-1}$. HRMS (ESI, $\mathrm{m} / z$ ): Calcd for $\mathrm{C}_{21} \mathrm{H}_{20} \mathrm{~N}_{3} \mathrm{O}_{3}[\mathrm{M}+\mathrm{H}]^{+} 362.1505$, found 362.1527

7-(3,4-Dimethylphenyl)-3,8,10,11-tetrahydropyrano[3,4-c]pyrazolo[4,3-f $]$ quinoline (4k). M.p $>300{ }^{\circ} \mathrm{C} ;{ }^{1} \mathrm{H}$ NMR $\left(\mathrm{DMSO}-d_{6}, 400 \mathrm{MHz}\right): \delta_{\mathrm{H}} 2.31\left(\mathrm{~s}, 6 \mathrm{H}, 2 \mathrm{CH}_{3}\right), 3.41\left(\mathrm{~s}, 2 \mathrm{H}, \mathrm{CH}_{2}\right), 4.15(\mathrm{t}, J$ $\left.5.6 \mathrm{~Hz}, 2 \mathrm{H}, \mathrm{CH}_{2}\right), 4.79$ (s, 2H, $\left.\mathrm{CH}_{2}\right), 7.25 \sim 7.31(\mathrm{~m}, 2 \mathrm{H}, \mathrm{ArH}), 7.37$ (s, 1H, ArH), 7.86 7.93 (m, 2H, ArH), 8.58 (s, 1H, CH), 13.72 (s, 1H, NH). IR (KBr): v3187, 3131, 3094, 3036, 2934, 2870, 1670, 1592, 1561, 1529, 1452, 1426, 1383, 1351, 1320, 1237, 1159, 1099, 1032, 983, 939, 918, 887, 825, 793, 735, $719 \mathrm{~cm}^{-1}$. HRMS (ESI, $\mathrm{m} / z$ ): Calcd for $\mathrm{C}_{21} \mathrm{H}_{20} \mathrm{~N}_{3} \mathrm{O}[\mathrm{M}+\mathrm{H}]^{+} 330.1606$, found 330.1627 .

7-Piperonyl-3,8,10,11-tetrahydropyrano[3,4-c]pyrazolo[4,3-f]quinoline (4I). Mp 289 290 ${ }^{\circ} \mathrm{C}$; ${ }^{1} \mathrm{H}$ NMR (DMSO- $\left.d_{6}, 400 \mathrm{MHz}\right): \delta_{\mathrm{H}} 3.41\left(\mathrm{~s}, 2 \mathrm{H}, \mathrm{CH}_{2}\right), 4.16\left(\mathrm{t}, J 5.6 \mathrm{~Hz}, 2 \mathrm{H}, \mathrm{CH}_{2}\right), 4.81(\mathrm{~s}, 2 \mathrm{H}$, $\mathrm{CH}_{2}$ ), 6.12 (s, 2H, CH$)_{2}, 7.03 \sim 7,07$ (m, 2H, ArH), 7.18 (s, 1H, ArH), 7.86 7.96 (m, 2H, ArH), 8.57 (s, 1H, CH), 13.72 (s, 1H, NH). IR (KBr): v 3191, 3159, 3107, 3050, 2932, 2872, 1606, 1588, 1571, 1530, 1500, 1438, 1377, 1351, 1327, 1254, 1233, 1180, 1125, 1088, 1039, 979, 953, 915, 883, 856, 816, 739, $725 \mathrm{~cm}^{-1}$. HRMS (ESI, $\mathrm{m} / z$ ): Calcd for $\mathrm{C}_{20} \mathrm{H}_{16} \mathrm{~N}_{3} \mathrm{O}[\mathrm{M}+\mathrm{H}]^{+} 346.1192$, found 346.1218 .

\section{Acknowledgements}

We are grateful to the National Natural Science foundation of China (20802061), the Priority Academic Program Development of Jiangsu Higher Education Institutions and Qing Lan Project (08QLT001, 10QLD008) of Jiangsu Education Committee for financial support.

\section{References}

1. (a) Yamada, N.; Kadowaki, S.; Takahashi, K.; Umezu, K. Biochem. Pharmacol. 1992, 44, 1211. (b) Faber, K.; Stueckler, H.; Kappe, T. J. Heterocyl. Chem. 1984, 21, 1177. (c) Johnson JV.; Rauckman, S.; Baccanari P. D.; Roth, B. J. Med. Chem. 1989, 32, 1942.

2. (a) Danel, A.; Gondek, E.; Kityk, I. Opt. Mater. 2009, 32, 267. (b) Khachatryan, K.; Boszczyk, W.; Tomasik, P. Polish J. Chem. 2005, 79, 1645. (c) Luszczynska, B.; Dobruchowska, E.; Glowacki, I.; Ulanski, J.; Jaiser, F.; Yang, X.; Neher, D.; Danel, A. J. Appl. Phy. 2006, 99, 024505/1. (d) Gondek, E.; Kityk, I. V.; Sanetra, J.; Szlachcic, P.; Armatys, P.; Wisla, A.; Danel, A. Opt. Laser Technol. 2006, 38, 487. Chem. Abstr. 2006, 145, 237438. (e) Gondek, E.; Kityk, I. V.; Danel, A.; Wisla, A.; Sanetra, J. Synth. Metals. 2006, 156, 1348. 
3. (a) Al-Qahtan, A.; Siddiqui, Y. M.; Bekhit, A. A.; El-Sayed, O. A.; Aboul-Enein, H. Y.; Al-Ahdal, M. N. Arch. Pharm. 2005, 338, 484. (b) Vartale, S. P.; Jadhav, J. S.; Kale, M. A.; Kuberkar, S. V. Indian J. Heterocycl. Chem. 2006, 16, 163.

4. Hays, D. S.; Prince, R. B.; Haraldson, C. A.; Bonk, J. D. PCT Int. Appl. WO 2006107851 A1 12 Oct 2006 152pp. Chem. Abstr. 2006, 145, 419133.

5. Merrill, B. A.; Danielson, M. E.; Hays, D. S.; Amos, D. T.; Heppner, P. D.; Kshirsagar, T. A.; Lundquist, G. D. Moser, W. H. PCT Int. Appl. WO 2006107771 A2 12 Oct 2006 135pp. Chem. Abstr. 2006, 145, 419140.

6. (a) Nagaiah, K.; Sreenu, D.; Srinivasa, Rao R.; Vashishta, G.; Yadav, J. S. Tetrahedron Lett. 2006, 47, 4409. (b) Lin, X. F.; Cui, S. L.; Wang, Y. G. Tetrahedron Lett. 2006, 47, 4509. (c) Maiti, G.; Kundu, P. Tetrahedron Lett. 2006, 47, 5733. (d) Vikram, G.; Rajagopal, N. Tetrahedron Lett. 2009, 50, 1243. (e) Li, Y. C.; Zhang, J. M.; Dong, L. T.; Yan, M. Chinese J. Chem. 2006, 24, 929. (f) Xia, M.; Lu, Y. D. Synlett 2005, 2357. (g) Rai, N. P.; Shashikanth, S.; Arunachalam, P. N. Synth. Commun. 2009, 39, 2125. (h) Kudale, A. A.; Kendall, J.; Miller, D. O.; Collins, J. L.; Bodwell, G. J. J. Org. Chem. 2008, 73, 8437.

7. (a) Malleshwar, D.; Gautami, K.; Jayashree, A. Org. Chem. An Indian J. 2009, 5, 344. (b) Mali, J. R.; Pratap, U. R.; Jawale, D. V.; Mane, R. A. Tetrahedron Lett. 2010, 51, 3980. (c) Tu, S. J.; Wu, S. S.; Zhang, X. H.; Han, Z. G.; Cao, X. D.; Hao, W. J. Synth. Commun. 2010, 40, 1057. (d) Duggineni, S.; Sawant, D.; Saha, B.; Kundu, B. Tetrahedron 2006, 62, 3228. (e) Jachak, M. N.; Avhale, A. B.; Medhane, V. J.; Toche, R. B. J. Heterocycl. Chem. 2006, 43, 1169.

8. (a) Cho, C. H.; Neuenswander, B.; Lushington, G. H.; Larock, R. C. J. Comb. Chem. 2009, 11, 900. (b) Zeng, L. H.; Cai, C. J. Comb. Chem. 2010, 12, 35. (c) Das, B.; Balasubramanyam, P.; Krishnaiah, M.; Veeranjaneyulu, B.; Reddy, G. C. J. Org. Chem. 2009, 74, 4393. (d) Wang, G. W.; Gao, J. Org. Lett. 2009, 11, 2385. (e) Mal, D.; De, S. R. Org. Lett. 2009, 11, 4398. (f) Parvatkar, P. T.; Parameswaran, P. S.; Tilve, S. G. J. Org. Chem. 2009, 74, 8369. (g) Madhusudana Reddy, M. B.; Nizam, A.; Pasha, M. A. Synth. Commun. 2010, 40, 3728. (h) Datta, B.; Madhusudana Reddy, M. B.; Pasha, M. A. Synth. Commun. 2011, 41, 2331. (i) Madhusudana Reddy, M. B.; Pasha, M. A. Synth. Commun. 2011, 41, 1875.

9. (a) Wang, X. S.; Wu, J. R.; Zhou, J.; Tu, S. J. J. Comb. Chem. 2009, 11, 1011. (b) Wang, X. S.; Yang, K.; Zhou, J.; Tu, S. J. J. Comb. Chem. 2010, 12, 417. (c) Wang, X. S.; Wu, J. R.; Li Q.; Yao, C. S.; Tu, S. J. Synlett 2008, 1185. (d) Wang, X. S.; Zhang, M. M.; Zeng, Z. S.; Shi, D. Q.; Tu, S. J.; Wei, X. Y.; Zong, Z. M. J. Heterocycl. Chem. 2008, 45, 71.

10. (a) Ji, S. J.; Wang, S. Y.; Zhang, Y.; Loh, T. P. Tetrahedron 2004, 60, 2051. (b) Lin, X. F.; Cui, S. L.; Wang, Y. G. Tetrahedron Lett 2006, 47, 3127. 